\title{
Does Endoscopic Assessment of Mucosal Healing Affect IBD Management?
}

\author{
Kian Keyashian
}

Received: 20 July 2014/ Accepted: 24 July 2014/Published online: 17 August 2014

(C) Springer Science+Business Media New York 2014

Diagnosis of ulcerative colitis (UC) or Crohn's disease (CD) relies on the combination of clinical, laboratory, and endoscopic data, which are needed for diagnosis according to adult and pediatric guidelines. Although the importance of an initial endoscopy is well documented, much less is known about the timing of and indications for follow-up endoscopy, with little stated in adult and pediatric guidelines and in the medical literature.

Guidelines from the American Society for Gastrointestinal Endoscopy (ASGE) published in 2008 questioned the utility of endoscopy in exacerbations of pediatric IBD responding to therapy, citing poorer quality of life and perceived excess risk in the pediatric population [1]. Nevertheless, these questions had little supporting evidence until 2009, when a cross-sectional cohort study based at Baylor University was published, in which 285 endoscopic procedures in 230 children with established IBD performed for gastrointestinal bleeding, abdominal pain, weight loss, and diarrhea were evaluated. The authors reported that $42 \%$ of procedures led to documented management changes such as a change of medication regimen, hospital admission, and surgical consultation [2]. Patients with CD (as compared to UC) and patients with mucosal injury (as compared to mucosal healing) were more likely to experience a management change with the initiation of medical therapy; of note, patient symptoms and laboratory values were not associated with a change in management. The authors concluded that endoscopic mucosal evaluation provided important independent data for the evaluation and management of pediatric IBD, particularly in CD.

K. Keyashian $(\bowtie)$

Oregon Health \& Science University, Portland, OR, USA

e-mail: keyashia@ohsu.edu
In the adult population, the value of repeated endoscopy is even less well studied. An early randomized clinical trial published by the GETAID group provided no data to support the value of colonoscopy in deciding the length of steroid treatment in colonic CD [3]. Of 136 patients in clinical remission for persistent disease, 96 with mucosal lesions were randomized to start a taper or continue prednisone at the same dose for 5 weeks before tapering. While the prolongation of prednisolone therapy improved endoscopic scores in that subgroup, the success of steroid weaning was almost identical between groups. Yet, in a parallel French study of 85 patients who underwent colonoscopy, 46 had severe and 39 had moderate endoscopic colitis, with response to medical therapy mostly observed in the latter group. [4].

In 2006, the ASGE reiterated an important finding from the literature: Subjective symptoms are not reliable indicators of disease severity, whereas endoscopy is an objective tool [5]. The technical review underlying the guidelines suggested that endoscopy may be helpful in predicting the need for intensified medical therapy or for surgical intervention. Nonetheless, no guidance is provided in the summary guidelines from the organization. The European Crohn's and Colitis Organisation (ECCO) guidelines support the need for repeat endoscopy if the diagnosis remains in doubt, but do not support routine endoscopy for patients in clinical remission unless it is likely to change management [6]. ECCO guides that endoscopic assessment is indicated in cases of relapse, refractoriness, new symptoms, or when surgery is being considered. Nevertheless, no investigator has attempted to determine whether the findings reported in the 2009 pediatric study are applicable in adults until recently.

In this issue of Digestive Diseases \& Sciences, Kim et al. [7] attempted to replicate the Baylor pediatric study in an 
adult population. In this retrospective observational study from two Korean tertiary hospitals, the investigators enrolled 188 patients with IBD—69 with CD and 119 with UC - with follow-up endoscopies in 130 patients ( 48 with $\mathrm{CD}$ and 82 with UC). Treatment changes were categorized as "changed aggressively," "changed lightly," "unchanged," and "changed to medication for other diseases." Treatment was unchanged in $68.8 \%$ of CD patients and in $65.9 \%$ of UC patients. Treatment plans were changed after follow-up endoscopic examination more frequently in the group with an endoscopic indication, which included "unexplained abdominal pain," "diarrhea," "hematochezia," "fever," "weight loss," and "checking short-term treatment response." In UC but not in $\mathrm{CD}$, such indications for colonoscopy were also predictive of hospitalization. In contrast with the pediatric study, the authors concluded that follow-up endoscopy might not have a significant impact on the overall clinical course and outcomes in patients with IBD.

With these results, the gastroenterologist may wonder what can be concluded from these two disparate articles on which somewhat contradictory guidelines are based. The recent study certainly has limitations brought up by the authors, including its small study population, retrospective design, and pre-biologic management. Moreover, IBD in the Korean population has some notable differences from that in the West, which could challenge the applicability of the adult study in the Western population; a 2010 study, for example, reported a possible better clinical response to therapy in UC with lower colectomy rate and more perianal disease with lower operation rate in CD in the Korean population [8]. Despite these shortcomings, Kim and colleagues are the first group to tackle this important question in the adult population and provide some evidence that a colonoscopy that truly has "no indication" may not be worthwhile.

The more important question that Kim and colleagues and their pediatric counterparts do not directly address is what the appropriate indications for colonoscopy should be. More specifically, in addressing the contribution of endoscopy to IBD management, should assessment of mucosal improvement or healing be a legitimate standalone indication for colonoscopy? Numerous studies have provided evidence that mucosal healing may be a better goal for treatment with respect to important outcomes (clinical remission, surgery, and colorectal cancer), but most of this evidence is retrospective or derived from subset analyses. In the article, Kim et al. [7] even suggest that a prospective study design would be difficult due to the observation that clinicians' therapeutic plans are based on the combination of endoscopy and other symptoms and signs.

In the last 1-2 years, a few studies have prospectively attempted to investigate a similar question. The Post Operative Crohn's Endoscopic Recurrence (POCER) study enrolled 174 patients with $\mathrm{CD}$, basing therapy after intestinal resection on the risk of recurrence [9]. Patients were randomized to colonoscopy at 6 months (independent of symptoms) versus no colonoscopy. The authors reported that, independent of which drug was chosen and how the risk of the patient was assessed, the only factor predictive of mucosal healing at 18 months was the 6 month colonoscopy. Another recent trial, REACT-1 (Randomized Evaluation of an Algorithm for Crohn's Treatment) is a cluster randomization trial comparing conventional sequential treatment $(\mathrm{CM})$ versus early combined immunosuppression (ECI) and algorithmic management in $\mathrm{CD}$ [10]. At 12 and 24 months, no difference was found in the primary end point, mean remission rates, between the ECI and CM groups. Nonetheless, remission was defined based purely on symptoms, using the Harvey-Bradshaw Index (HBI). With this prospective evidence, the same research group is now tackling REACT-2, a cluster randomized controlled trial of an enhanced treatment algorithm versus a conventional symptom-based algorithm for CD in the USA and Canada [11]. The enhanced treatment algorithm features early use of combined antimetabolite-biologic therapy, with treatment intensification based on ileocolonoscopic findings; this is in contrast to the conventional "step-care" algorithm, which bases treatment escalation on symptoms. The investigators plan to report the complications of $\mathrm{CD}$ at 6 and 12 months.

In conclusion, Kim and colleagues demonstrate that a follow-up colonoscopy in IBD with "no indication" is unlikely to alter a patient's clinical outcomes. Still, with a promising prospective study utilizing colonoscopy in clinical decision making underway, the new indication for follow-up colonoscopy of "assessment of mucosal response/healing" will likely positively impact important outcomes.

\section{References}

1. Lee KK, Anderson MA, Baron TH, et al. Modifications in endoscopic practice for pediatric patients. Gastrointest Endosc. 2008;67:1-9.

2. Thakkar K, Lucia CJ, Ferry GD, et al. Repeat endoscopy affects patient management in pediatric inflammatory bowel disease. Am J Gastroenterol. 2009;104:722-727.

3. Landi B, Anh TN, Cortot A, et al. Endoscopic monitoring of Crohn's disease treatment: a prospective, randomized clinical trial, the Groupe d'Etudes Therapeutiques des Affections Inflammatoires Digestives. Gastroenterology. 1992;102:1647-1653.

4. Carbonnel F, Lavergne A, Lemann M, et al. Colonoscopy of acute colitis: a safe and reliable tool for assessment of severity. Dig Dis Sci. 1994;39:1550-1557.

5. Leighton JA, Shen B, Baron TH, et al. ASGE guideline: endoscopy in the diagnosis and treatment of inflammatory bowel disease. Gastrointest Endosc. 2006;63:558-565. 
6. Annese V, Daperno M, Rutter MD, et al. European evidence based consensus for endoscopy in inflammatory bowel disease. $J$ Crohns Colitis. 2013;7:982-1018.

7. Kim DH, Park JJ, Park SJ, et al. Effect of follow-up endoscopy on the outcomes of patients with inflammatory bowel disease. Dig Dis Sci. (Epub ahead of print). doi:10.1007/s10620-014-3197-0.

8. Kim SK, Kim WH. Inflammatory bowel disease in Korea: epidemiological, genomic, clinical, and therapeutic characteristics. Gut Liver. 2010;4:1-14.

9. De Cruz P, Kamm MA, Hamilton AL, et al. Optimising postoperative Crohn's disease management: best drug therapy alone versus colonoscopic monitoring with treatment step-up. The POCER study. Gastroenterology. 2014;146:S-174.

10. Khanna R, Levesque BG, Bressler B, et al. Early combined immunosuppression for the management of Crohn's disease: a community-based cluster randomized trial. Gastroenterology. 2014;146:S-187.

11. National Institute of Health; AbbVie. Enhanced Algorithm for Crohn's Treatment Incorporating Early Combination Therapy. ClinicalTrials.gov. Bethesda, MD: National Library of Medicine (US); 2000. (Cited 30.05.2014). http://clinicaltrials.gov/show/ NCT01698307. NLM Identifier: NCT01698307. 